\title{
EL ESTUDIO DE LA ESTRUCTURA SOCIAL DESDE LA PERSPECTIVA PROCESAL
}

\author{
José A. Gatmendia \\ (Universidad de Málaga)
}

Los análisis estructural y fincional no son bloques cerrados sino que pueden existir distintas interpretaciones. La práctica conservadora de ciertos enfoques del análisis funcion:l (o estructural) no implica necesariamente que sólo pueda ser utilizado en este sentido. «Estructura» y «cambio» no son conceptos contradictorios ccrmo tampoco lo son «estabilidad» y «cam * bio». El análisis de la estructu.a social a partir de la profundización en los procesos desartollados en esie artículo es una superación de las teotías clásicas partiendo precisamente del legado funcionalista y estructuralista. 
La acusación más repetida contra el funcionalismo es probablemente la de su incapacidad para explic ar el cambio con la consiguiente tendencia inevitable al conservadurismo. J'ambién el estructuralismo ha sido ocasionalmente blanco de parecidos atc ques, aunque no todo estructuralismo haya sido tratado del mismo modo. (luiero mostrar el grado de verdad de tales acusaciones, al mismo tiempo qre sus inexactitudes a causa de la ambigüe dad definitoria del funcionalismo y del estructuralismo, la cual debe ser clatificada desde la perspectiva sistémico-procesal.

El enfoque procesal ha ido aproximándose gradualmente al análisis de sistemas, tratando el problema del cambio de un modo peculiar: es decir, los sistemas sociales apaticen como elaboradores de sus propias estructuras. La concepción de la sociedad como sistema abierto, en el que la acción social se explica sobre la base de múltiples interacciones (mejor, retroalimentaciones) entre las fartes y con ei Umwelt (entorno), podría incluso encajar el marxismo si se parte de la teoría de que la infraestructura económica determina en ultima instancia el cuadro de retroalimentaciones.

A continuación me ocupar: de un ejemplo clásico, ilustrativo de las difetencias teóricas y metodológicas del funcionalismo establecido respecto de otros enfoques, basados inclıso en el análisis funcional. Ello dará pie a la exposición de las variedades de análisis funcional y estructural (estructuralista), de la explicación del cambio sobre la base del análisis de sistemas, y de los procesos de instit acionalización.

Funcionalismo tradicional y tratamiento de los procesos sociales: Un ejemplo clásico

Muchas de las acusaciones lanzadas contra el funcionalismo se tefie ren a la presunta identificación, más o menos explícita, de funciones y recesidades. Consecuentemente, entre funcionamiento y estructura. Tales inconvenientes parecen persistir en la práctica, pese al recurso último de 
las funciones latentes, según trataré de mostrarlo en seguida a propósito del caso de E. Durkheim.

Durkheim hizo una importante distinción entre funciones manifiestas y funciones latentes, como lo hiciera más tarde Merton, aunque utilizando otro vocabulario. Apuntó también a los inconvenientes de quedarse en una explicación funcional de los hechos sin recurrir también a la inquisición de la «causa eficiente». ${ }^{1}$

Por otra parte, existen en la producción durkheimiana importantes momentos relacionados con el conflicto y el poder, que necesariamente invitan al sociólogo a cuestionar la fácil equiparación funcionalista de funciones y estructuras. Así, el sociólogo francés subrayó la carga conflictiva que supone la marcha progresiva de la división del trabajo y la consiguiente amenaza del proceso de burocratización. ${ }^{2}$ Con todo, no parece fácil reducir aquella producción a una condición no funcionalista, como lo ha intentado alguien. Albert Pierce, por ejemplo, ha tratado de demostrar esta circunstancia, aunque, en mi opinión, sin éxito. Pierce se empeña en descubrir en el sociólogo francés la ausencia de un inconveniente típico del funcionalismo: «La amplia tendencia moderna a equiparar "Eunciones" $\mathrm{y}$ "necesidades", mientras que en Durkheim es innegable la aguda distinción entre ambas.» Con tal propósito trae a colación aquel principio que figura en las primeras páginas de La División del Trabajo Social: «En ptiner lugar, habremos de buscar cuál es la función de la división del trabajo: es decir, a qué necesidad corresponde.» Más aún (como afirmará en su siguiente gran obra): «Preguntarse cuál sea la función de la división del trabajo es [...] buscar a qué necesidad corresponde.»

Se habla, pues -observa A. Pierce-, de «correspondencia» y no de «equivalencia» o «ecuación», como erróneamente expresan las małas traducciones del francés al inglés. La argumentación parece más convincente todavía, de tenerse en cuenta otra conocida afirmación: "Sobre este asunto se suele razonar como si, en un organismo sano, cada detalle [...] tuviese un papel útil para su desempeño; como si cada estado interno respondiese exactamente a alguna condición externa y, por consiguiente, contribuyeta a asegurar, por su parte, el equilibrio vital.» ${ }^{4}$ Poco después

1. E. Durkheim, Las reglas del método sociológico (Buenos Aires: La Pleyade, $1970)$, p. 139.

2. E. Allardt, «E. Durkhein: Sein Beitrag zur Politischen Soziologie», Koelner Zeitscbrift fuer Soziologie und Sozialpsychologie (1968): 1.

3. A. Picrce, «Durkheim and Functionalism», en Kurt H. Wolff, Emile Duskheim

(Ohio: Ohio State University Press, 1960).

4. E. Dutkheim, op. cit., p. 51 . 
apunta: «Es legítimo suponer que ciertos arteglos anatómicos o funcionales no sirven directamente a nada.» (El énfasis de los adverbios «exactanente» y «directamente» utilizados por Durkheim, es mío.)

Se ve, pues, cómo tales proposiciones encajan en los propósitos encaminados a demostrar la condivión no funcionalista: en este caso la presunta distinción conceptual entre funciones y necesidades, funcionamiento y estructura. Sin embargo, habre nos de preguntarnos acerca del por qué del vocablo «directamente». $\mathrm{E}_{n}$ efec $\mathrm{o}$, «indirectamente» ¿podrán aquellos arteglos servir de algo? ¿Podrá habiarse entonces de la ecuación - no sólo cotrespondencia - de necesidades y funciones? El término «indirectamente» cabe ser enfocado muy bien desde una perspectiva de vocabulario ya clásico. En efecto, aludiría a lo que Mktton designó por «funciones latentes». El crimen, el comportamiento desviado tienen la función indirecta (latente) de conseguir precisamente la armonía funciones-necesidades del sistema. «Indirectamente» establece Durl heim la correspondencia «exacta» entre el estado interno (condiciones, necesidades, estructuras) y la condición externa (funciones). Es la ecuación ratificada por la demostración del carácter estructural de la desviación siempre que se respete la invariancia estructural, que, por to mismo, es sólo rela ivamente (limitadamente) modificable. De lo contrario, el diagnóstico reza «anomía» o amenaza grave al sistema mismo.

Se presupone, pues, la ma cha conjunta de funciones y necesidades, aunque sea bajo la concesión de ciertas perturbaciones hemeostáticas: es decir, asimilables por los umbrates de reducida variabilidad del sistema. Cuando aquella marcha conjunta aparece insostenible, es que ha irrumpido la anomía en el castillo inexpugnable del funcionalismo, es que simplemente "entonces no hay división del trabajo». ${ }^{5}$ Se comprende así que el intento de explicación causal, tan reclamado por el sociólogo francés, la misma separación causa-función deban crestionarse sariamente. En efecto, si el estudio de las funciones ha de constituir un instrumental para la explicación causal, ¿qué sucederá al pretenclerse averiguar las causas de un fenómeno - la anomía (por ejemplo, en su versión de lucha de clases) - por la maníaca atención a las funciones que, según la particular visión (conservadora quizás) del autor, no cumple? El funcionalismo teleológico clásico o el juicio de valor se han introducido por la puerta falsa.

Se comprende, pues, el tratamiento durkheimiano (parsoniano, etc.), del conflicto como fenómeno furdamentalmente «accidental» - por su condición de institucionalizado- $\mathrm{y}$, de rebasarse ciertos límites, patológico.

5. E. Durtcheim, De la division du travail social (París: Lacan, 1893), p. 396. 
Sin embargo, teniéndose en cuenta la reiterada y constante periodicidad de los mismos conflictos -el mismo Durkheim habla en tal sentido, al mencionar el estado «crónico» en que «lamentablemente» se encontraban¿por qué no presumir la posibilidad de su carácter estructural: es decit, estructural a la misma diferenciación funcional o, lo que es lo mismo, la expresa equiparación estructura-conflicto, estructura-proceso, etc?

En el esquema durkheimiano, la acción social discurre entre dos polos: integración funcional y anomía (disfuncionalidad), "desdiferenciación» social. La constante amenaza del diagnóstico de anomía implica la relativización, en el sentido de descubrir funcionalidad en muchos cambios «bruscos». Este adjetivo viene a significar «no espontáneo», asistémico o extra-sistémico. Se parte, pues, de la consideración de que está desprovisto de grandes problemas o graves perturbaciones. Sin embargo, se habla de crisis «agudas» y de que existe una esfera de la vida social donde la anomía se encuentra actualmente en estado «crónico». ${ }^{6} \mathrm{La}$ inmediata vecindad de los términos «actualmente» y «crónico» constituye un claro síntoma de la crítica situación que atravesaba un análisis sistémico más bien cerrado en la difícil tarea de explicación del cambio y del conflicto.

Se observa claramente el manejo durkheimiano de un modelo de sistema regido por procesos de retroalimentación hemeostáticos. El sistema es sólo relativamente abierto, dominando los mecanismos de autorregulación o compensación, más o menos espontáneos, de perturbaciones (crisis «normales», «felices»). Cuando tal autorregulación no se da, el sistema agoniza anómicamente.

Con todo, aunque la metodología peculiar (peculiat por su trasfondo teórico) seleccionada por Durkheim encaja en su marco teórico conservador, la metodología funcional (que enfoca la sociedad como sistema abierto) no es necesariamente conservadora. Así, el análisis funcional no es ajeno al marxismo mismo, según ha observado A. Stinchcombe.? Marx explicó repetidas veces la existencia (más que la estructura) de una institución en función de la contribución (positiva/negativa) a los intereses y jerarquía de poder de la estructura de clases. Así, el parlamentarismo «burgués» sólo podría imponerse cuando se reunieran las condiciones de funcionalidad respecto de los estados meta de una clase social (la burguesa), y de existencia de relaciones de poder favorables a dicha clase. Por ello mismo, el

6. E. Dutkheim, El sticidio (Buenos Aires: Schapite, 1969), p. 203.

7. A. L. Stinchcombe, Constructing Social Theortes (Nueva York: Hatcourt Brace, 1968), pp. 93 y ss. 
parlamentarismo «burgués» no ie dio en modos de producción diferentes al capitalista. Naturalmente, Masx manejó una estrategia funcional sobre el trasfondo del traterialismo histó:ico, siendo el modo de producción el marco explicativo último del cuadro de funciones.

Como se ve, el análisis funcional no es en sí conservador, aunque se dé tal tendencia por basarse fundarr entalmente en el estudio de las consecuencias, statu quo u operaciones-resultado de una estructura, las cuales actúan como causa de la explicación. Puede, sin embargo, ser conservador incluso en un contexto señaladamente ditıámico y hasta revolucionario: por ejemplo, ¿hasta qué punto se han tenido en cuenta los efectos disfuncionales (y por lo mismo, destructores) del parlémentarismo respecto del sistema establecido y capitalista? Por otra parte, no debe olvidarse que el carácter conserva dor, reformador, tevolucionatio, etc., del análisis funcional dependerá de la definición de los estados-meta (que pueden ser hasta utópicos) en función de los cuales se apreciará la contrbución (positiva/negativa) de los hechos sociales.

La práctica conservadora del análisis funcional (en buena medida, por encontrarse atada a una estructura social que se resiste al análisis profundo) ha divulgado una imagen conservadora del mismo, que reflejaría una fatal incapacidad de explicación de los procesos sociales. Sin embargo, hay una gran variedad de funcionalismos y estructuralismos, que tratan también de tnuy diversa forma el capítulo del cambio.

\section{Variedades de análisis estructural y funcional: convergencias $y$ divergencias}

Muchas explicaciones funcionales han sido deficientes científicamente a causa de la imprecisión definitıria de términos claves, tales como «adaptación», «integración», «supervivencia», etc. $\mathrm{El}$ esquema funcionalista habría sido más útil, de renunciarse a establecer a priori, sin previa justificación teórica, el sentido de cunceptos mencionados o la lista de requisitos y alternativas, especificando, en cada caso, las significaciones correspondientes: por ejemplo, «adapłación» pero, ¿para qué grupos? A partir de una previa justificación teórica puede darse una mayor colaboración entre análisis causal (aunque sıa entendido tradicionalmente: es decir, linealmente) y análisis funcional. Éste siempre será, por otra parte, útil, aunque sólo sea por esclarecer las relaciones entre elementos (funcionalismo relacional), funciones respecto de $G$ (propiedades del sistema), listas de requisitos considetados más o menos importantes (una nueva versión de las alternativas funcionales), etc. Teniendo en cuenta las apuntadas dif- 
cultades, señaladas por Francesca M. Cancian, han ido apareciendo paradigmas de análisis alternativos reseñados por la misma autora: ${ }^{8}$ es decir, inversión de las explicaciones funcionales en beneficio de las causales, reducción del alcance, demasiado abstracto y consecuentemente de escaso poder explicativo de las proposiciones funcionales clásicas, introducción de modelos de retroalimentación de variables, ${ }^{9}$ que supondxía un nuevo enfoque del análisis de la estructura y del cambio, ${ }^{10}$ no concebible ya al modo esencialista tradicional de simple «transustancialización de esencias».

$E_{n}$ efecto, la sociedad se concibe como sistema o conjunto interrelacionado de elementos, que, a su vez, se interrelaciona con todo lo demás: es decir, el entorno o ambiente. Interesa analizar su estructura o el nodo de organización de las partes y con el entorno, librándose series de retroalimentaciones procesadoras o generadotas de nuevas estructuras. Se trata, pues, de una estrategia estructural diacrónica (el tiempo no es un peligro sino elemento constitutivo de la estructura del sistema) y transformacional (necesidad de interrelación dialéctica y retroalimentación de elementos para la solución de problemas y contradicciones). " Xa no se ve la sociedad como una «sustancia» o sistema cerrado y prácticamente inmóvil sobre el que acontecen (la historia sería fundamentalmente acontecimiento, accidente) accidentes diversos: por ejemplo, el cambio. Al desprenderse de esta estra. tegia fundamental, el análisis funcional —basándose en modelos de análisis mecánicos y biológicos, cibernéticos- experimentó un cambio radical en el modo de estudiar la sociedad. El estructuralismo y el funcionalismo subrayan la organización. El axioma «el todo es más que la suma de las partes» — siendo el más que precisamente la organización- refleja la sustitución del interés en la sustantividad por la considexación sisténicoestructural del objeto. El ser de éste, del todo, se explica por su organización: en esto se diferencia de la simple suma de las partes. Las diferencias «sustanciales» entre «todos» se explican por su diferente organización, tanto interna como en relación con el entorno. La consideración organizacional y relacional se encuentra presente en cualquier análisis funcional, aunque el funcionalismo tradicional la haya viciado de fuertes residuos esencialistas y sustancializantes.

Los primeros pasos del análisis funcional o del funcionalismo tradicio-

8. F. M. Cancian, «Varieties of functional analysis», International Encyclopedia of the Social Sciences, vol. IV, p. 37.

9. E. Nagel, La logica sin metafisica (Madrid: Tecnos, 1962), pp. 240 y ss.

10. F. M. Cancian, op. cit., p. 39.

11. W. B. Buehl, Struktur und Funktion (Munich: NTW, 1973), p. 11. 
nal (Malinowski, Radcliffe-13rovn, Durkheim, el mismo Lévi-Strauss) no logran liberarse del lastre sustarcialista, como lo prueban los famosos pos* tulados del análisis funcional (1nidad, universalismo, indispensabilidad y reciprocidad) por los que «todo» funciona «para todo» y de modo más bien recíptoco y simétrico. Es un todo «abstracto» e indiferenciado cuya organización no se analiza, pest: a que, claro está, una institución puede ser funcional a una parte de açuel todo o a un determinado tipo de su organización y disfuncional tesp zcto de otras partes. Se descuida, pues, el aspecto relacional en favor del e foque sustancialista y burdamente holista. Por Io mismo, la estructura institucional y la misma estructura social no se analizan como resultado procesal y siempre efímero de interacciones y retroalimentaciones (conflictos, luchas de clases, desviaciones). Todo lo más, se admiten mecanismos de: retroalimentación relativamente abiertos (homeostáticos), que «toleran» cambios modestos entre umbrales de invariancia limitados. La otra salida es simplemente la destrucción del sistema, la anomía. En el caso del mismor Lévi-Strauss, su estructuralismo de corte fundamentalmente sincrónico y de oposición binaria -a diferencia del estructuralismo más bien genétizo y diacrónico de J. Piaget y L. Goldman-12 queda anquilosado en le. simetría: "Como consecuencia de la tesis de la reciprocidad fundamental se pierde de vista todo lo que interesa a sociólogos y antropólogos de la cultura: es decir, la desviación respecto de las reglas de matrimonio (al parecer, sólo de carácter prescriptivo), las complicaciones del proceso de ir.tercambio, el afianzamiento de asimetrías a lo largo de prolongadas secuer cias, la complejidad del orden social y la invasión, incluso exterminio, de la ordenación matrimonial por factores políticos y económicos.» ${ }^{13}$

Con ello, Lévi-Strauss «no differe mucho de la posición en sociología de la primitiva escuela estructuril funcionalista que se asignaba cono objetivo la búsqueda de ciertos pterrequisitos funcionales universales de la sociedad». ${ }^{14}$ Distanciándose progresivamente de la preocupación por la simetría y de la voluntad de descubrir «la» estructura del orden universal (y del cerebro mismo), otros han dedicado sus esfuerzos al estudio de «epistemes» 0 formas de inteligibilidad de cada época, ${ }^{15}$ pluralidad de prácticas, etc. Tal pluralidad (también de niveles, regiones, etc.), de la estructura aparece en un discípulo de M. Foucauit, L. Aithusser, que, dentro del marxismo, interpreta el materialismo histótico desde perspectivas es-

12. Ibid., pp. 38 y ss.

13. Ibid., p. 41.

14. T. Bottomore, La sociologia :narxista (Madrid: Alianza, 1976), p. 94.

15. M. Foucault, Las palabras y las cosas (México: Siglo XXI, 1961). 
tructuralistas, formalistas y más o menos anhistóricas y deterministas, ${ }^{16}$ continuadas en M. Godelier, E. Balibar y N. Poulantzas. En todos ellos juega un importante papel el concepto de «estructura a dominante», que «define la totalidad marxista como un todo complejo que posee la unidad de una estructura articulada, en la que existe un elemento que desempeña el papel dominante y otros que le están subordinados; unidad dinámica en la que hay un intercambio de papeles, siendo el nivel económico el que determina en última instancia el elemento de la estructura social que desem. peñará el papel dominante». ${ }^{17}$ Así, pues, los procesos y retroalimentaciones de la estructura obedecen, en última instancia, a las relaciones de producción, aunque, por el carácter sobredeterminante de las contradicciones, un nivel (por ejemplo, el ideológico o el político-jurídico) puede afectar y dominar incluso los procesos de estructuración y reestructuración. Esa instancia última y fundamental, que para Althusser está representada por las relaciones de producción, parece diluirse en $N$. Poulantzas y otros en la articulación misma de las partes del sistema, la cual se constituitía en auténtica matriz de un modo de producción: «lo que distingue un modo de producción y, consecuentemente, especifica al mismo en esa forma particulat de articulación que mantienen sus niveles: es lo que se designaxá en lo sucesivo como matriz de un modo de produccións. ${ }^{18}$ Con ello se privilegiaría la combinación frente al contenido, la sintaxis frente a la semántica, y el hombre corre peligro de dejar de ser protagonista de la historia, ${ }^{19}$ te flejándose así el impacto de la sociedad programada y burocratizada. ${ }^{20}$

Así, pues, frente a la tendencia rectificadora del funcionalismo clásico, privilegiando el estudio de las consecuencias «establecidas»-las cuales actuarían como causas explicativas de los fenómenos sociales-, el estructuralismo parece habetse inclinado hacia el formalismo y la combinatoria. Sin embargo, ambos convergen no sólo en llamativas deficiencias (respecto de la explicación del cambio: sobre todo, en el funcionalismo tradicional y en el estructuralismo sincrónico de oposición binatia) sino fundamentalmente en su común perspectiva relacional. Ambos se despegan građualmente de posiciones claramente especulativas y esencialistas en beneficio

16. L. Althusser, La revolución teórica de Marx (México: Siglo XXI, 1969); con E. Balibar, Para leer El Capital (México: Siglo XXI, 1974).

17. L. Althusser, La revolución tebrica de Marx (México: Siglo XXI, 1967), p. 166 .

18. N. Poulantzas, Poder politico y clases sociales en el Estado capitalista (Madrid: Siglo XXI, 1972), p. 6.

19. E. Mandel et al., Contra Althusser (Barcelona: Madrágora, 1974), pp. 39 y ss.

20. C. Moya, «Sobre la actualidad del sujeto», Sistema (octubre 1973). 
de un estudio sistémtco de la sociedad. Los sistemas socio-culturales tienen en común con los biológicos y físicos su fundamental condición de sistemas, diferenciándose de éstos for su peculiar modo de organización.

El estudio de la sociedad en cuanto sistema abierto equivale a su consideración como radicalmente dependiente del «entorno»: es decit, de otros sistemas (biológico, psíquico, etc.), de la sociedad global, etc. Esta originaria e inevitable interrelación entre las partes del sistema y de éste respecto del ambiente o entornc supone una nutrida red de retroatimentaciones que regulan (corrigen, tansforman, etc.) los procesos de estructuración y reestructuración del sistema considerado. El mismo tiene el "propósito" de reducir la complejidad o incertidumbre del entorno, en el que necesariamente está inmerso, seleccionando entre alternativas diversas: es decir, informándose o tomandı decisiones según la cantidad de información (bits), de acuerdo con la fórtmula de Shannon (réplica de la de entropía en física): $\mathrm{I}=\mathrm{N} I \mathrm{~h}_{2} \mathrm{~h}$, donde $\mathrm{I}$ es información; $\mathrm{N}$, número de elecciones (binarias); $h$, símbolos. Cuanto más diferenciado funcional-estructuralmente se encuentre un sistema socio-yultural, tanto mayor será su riqueza y variedad $\mathrm{y}$, por lo mismo, tantc más copiosas sus posibilidades de información y comunicación. En ésta las señales son signos, que tecogen el arsenal de elementos del universi simbólico y las connotaciones que receptor y emisor confieren desde códigos más o menos compartidos a los mensajes.

La apuntada riqueza de los sistemas socio-culturales - -sobre todo, en los más diferenciados y, por lo ruismo, más dependientes de un «intercambio genetalizado»- contrasta con la rigidez o «construcción» de los sistemas cerrados o los limitadamente: abiertos. Los sistemas cerrados contienen $a b$ initio una programación fija y prácticamente invariable frente a las perturbaciones externas, aunque quippan algunas variaciones o elasticidad: por ejemplo, la propia del termostato. El mismo cuerpo humano reacciona frente a las oscilaciones de temperatura ambiental manteniendo una temperatura interna relativamente constante, a diferencia de lo que suele ocurrir con una piedra, por ejemplo, que registra una variación de temperatura paralela a la del entorno.

Las diferencias apuntadas :ie han expresado también indicando que los sistemas cerrados (físicos y biológicos, aunque se reconozca en estos últimos un alto grado de "plasticidad») son, a diferencia de los abiertos, ricos en energia y pobres en información. Los primetos procesan fundamentalmente energía y los segundos, información: así, «una persona que habla un lenguaje extranjero a su acompañante emite -a juicio de su interlocutor- meros ruidos o energía vibratoria, porque este último no 
encuentra correspondencia entre la variedad estructurada de la energía vocal y el repertorio de sonidos significativos estructurados. Pero si comprende dicho lenguaje, y la información transmitida fuese: Cuidado, se nos viene encima un automóvil, esa ínfima proporción de energía vocal desencadenaría una gran cantidad de energía en quien actúa como receptor»."21

Se comprende, pues, que los sistemas abiertos se reestructuren continuamente al estar dominados fundamentalmente por el flujo de información, que, en los sistemas socio-culturales, se concibe como proceso de comunicación de signos (1a lengua, objetos de consumo, etc.). Así, la estructura del sistema «evoluciona» procesando información. La estructura es proceso porque el sistema replantea continuamente su organización: es decir, su suerte no depende sólo de los estados originarios sino de las perturbaciones del entorno. Está condenado a teadaptarse continuamente a nuevos desafíos, reducir incertidumbre, informarse: o sea, a poner orden en medio de la «complejidad caótica» circundante. Los sistemas socio-culturales no soportarían, pues, lo aleatorio o entrópico, a diferencia de lo que ocurre en los sistemas cerrados. Éstos tienden al «estado» de equilibrio, el cual deberá modificarse de algún modo si ha de trabajar el sistema. Precisamente, «el biólogo - a diferencia del físico, ocupado del estudio de sistemas fundamentales cerrados- toma buena nota de la existencia de gradientes de noequilibrio en los organismos vivos y quiere saber cómo se mantienen (se entiende, en desequilibrio), en contra de los impulsos de los sistemas físicos al estado de equilibrio. La respuesta reside en la fundamental diferencia entre los llamados sistemas cerrados y abiertos». ${ }^{22}$ Los sistemas abiertos no parecen seguir, pues, la segunda ley de la termodinámica, que afirma la tendencia de un sistema a alcanzar un estado de equilibrio o de máximo otden aleatorio y fortuito (o sea, desordenado o entrópico).

En realidad, todo puede tratarse cientiffcamente, lo vivo y lo no vivo, ${ }^{23}$ desde la perspectiva común del sistema, la cual reclama una metodología diferente a la de la mecánica clásica, considerada tradicionalmente como prototípica. La teoría general de los sistemas intenta precisamente atacar de modo directo la complejidad caótica u organizada -como un sistema de variables interactuantes $y$, en el caso de los sistemas abiertos,

21. W. Buckley, La sociologia y la teoría moderna de los sistemas (Buenos Aites: Amorrortu, 1973), p. 79.

22. Anatol Rapoport, «Foreword», en W. Buckley: Modern Systems Research for the Behavioral Sciences (Chicago: Aldine, 1974), p. 61.

23. Ibid., p. 20. 
dependientes en su desarrollo de las perturbaciones del entorno: «La cien. cia clásica se ocupó de aspectos de causalidad lineal (problema de dos variables, fundamentalmente) o de complejidad no organizada, manejable por métodos estadísticos y basados, en última instancia, en el segundo principio de la termodinámica. Sin tmbargo, en la biología y en la física moderna, los problemas de complejidad organizada o de interacción entre un amplio, aunque no infinito, núnero de variables, reclama el uso de un nuevo instrumental conceptual.y ${ }^{24}$

Por su relevancia (por ejerplo, en relación con la butoctacia y el poder) y su estrecha dependencia de la teoría general de los sistemas quiero referirme, a título ilustrativo, a la teoría de la organización. ${ }^{25}$ En el matco de la teotía de los sistemas, se enfocan las organizaciones como sistemas parciales, estudiándolos en relación con el entorno (así, otras organizaciones). Desde una perspectiva sistémica general, se relacionan los elementos destacados por la teoría clásica (racionalidad, estructura formal, intereses empresatiales y del personal, etc.), y por la escuela de relaciones humanas (irracionalidad, espontaneidad, $\epsilon$ structura informal), incluyéndose tanto la fábrica como el hospital, los partidos políticos, las cárceles, lo cual apunta a la interdisciplinariedad del arálisis de sistemas y, concretamente, de la organización. Es curioso observar, por otra parte, las críticas lanzadas contra la teoría moderna de la org unización ya que las mismas recuerdan las ya conocidas respecto de la teo:ía de los sistemas, en general.

Fundamentalmente, se ha reprochado a la teoría de la organización un enfoque anhistórico y demasiado generalizante, no recogiendo satisfactoriamente la «irrepetibilidad» (Einmaligkeit) de una organización (ei «individuo histótico» de Max Weker, por ejemplo), dado que las diferencias entre las distintas organizaciones pueden ser más decisivas que las supuestas semejanzas descubiertas teóricamente: en efecto, «cabe dudar de la posibilidad de una teoria (integrada) de la organización que articulara un sistema lógicamente consistente de proposiciones generales del tipo "si... entonces" $"{ }^{26}$ Por lo mismo, Renate Mayntz recomienda ocuparse, en primer lugar, de la selección de propisdades de la organización (tamaño, objetivos) que puedan considerarse especialmente expuestas a retroalimentación

24. Ludwig von Bertalanffy, «General System Theory: A Critical Review», General Systems, vol. VII (1962), tp. 1-20.

25. José A. Garmendia, «Teorí 1 de la organización», en S. del Campo, J. F. Marsai, y J. A. Garmendia, Diccionari) de Ciencias Sociales (Madrid: IEP/UNESCO, 1976), vol. II.

26. R. Mayntz, «Soziologie ker Organisations, R. Koenig: Handbucb der Empiriscben Sozidl Foroschung (Stuttgart: Enke, 1969), vol. II, p. 458. 
respecto a un determinado Umwelt, iguaimente relevante (otra organización la sociedad global).

Pese a las dificultades de una teoría general, la moderna teoría de la organización ha abierto, con todo, prometedoras perspectivas a partir del desarrollo de teorias de medio alcance, que han incluido sistemáticamente, junto a los elementos de «racionalidad burocrática» los momentos noracionales. Así, H. A. Simon ${ }^{27}$ corrigió el modelo tradicional de la teoría de las decisiones desde el supuesto de la "racionalidad limitada», afitmando que los actores no persiguen tanto la maximización calculada de los beneficios y recompensas, sino resultados satisfactorios. Al incluir simultáneamente en sus modelos diversas variables en interacción, la investigación se ha movido, aunque sea implícitamente, en el marco de la teoría de los sistemas, subrayándose de entrada los elementos disfuncionales del fenómeno burocrático, ${ }^{28}$ el grado de buroctatización en función de factores tales como inestabilidad del mercado ${ }^{29}$ o, en fin, la misma organización como refejo de un modelo «natural» más que «racional», según la distinción de Gouldner: es decir, se la analiza tal como realmente se estructura, en lugar de limitarse al estudio clásico y weberiano de la relación tecursosobjetivos.

\section{Estructura social y desviación}

Por lo apuntado se observa un intento de superación de modelos organizacionales mecánico-racionales (Pareto, el mismo Max Weber, para quien el «sentido» de la acción es fundamentalmente racional-burocrático, E. Durkheim y su modelo de solidaridad mecánica) y oxgánicos (organicismo de Spencer, equilibrio homeostático, etc.). En general, los modelos basados en analogías han tendido a "sustancializar» la sociedad y, por ello, a cerrarse a la posibilidad de explicar el cambio estructural. En este sentido, «T. Parsons arguye correctamente que, para estudiar el cambio social, es necesario algún punto de referencia a partit del cual podarnos dar por producido el cambio. Pero luego quiere la fijación de una estructura [...] que se convierte en la "estructura", apareciendo el cambio y la desviación como algo residuals. ${ }^{30}$

27. H. A. Simon, Administrative Bebayior (Nueva York: 1945).

28. M. Crozier, El fenómeno burocrático (Buenos Aires: Amorrortu, 1974).

29. A. L. Stinchcombe, «Bureaucratic and craft administration of production: A comparative study», Administrative Science Quarterly, vol. IV (1960).

30. W. Buckley, op. cit., pp. 26-33. 
Criticando el funcionalismo, N. Luhmann observa que el problema (Ia desviación) es algo constitutivo de la organización (del sistema y de sus relaciones con el entorno), «de forma que la estabilidad no debe comprenderse ya como sustancia invariante sino como relación sistema-entorno o invariancia relativa de la estructura y de los límites det sistema [...] sin que pueda determinarse inequírocamente cuándo aparece un nuevo sistema». ${ }^{31}$ Precisamente, el princijjio de no contradicción mismo no parece encajar en el enfoque sistémicc, desde cuya perspectiva la perturbación (problema, contradicción) es al; ‘o constitutivo de los sistemas que mediante ella elaboran y reelaboran su estructura en un continuado proceso. Esto parece aplicarse hasta a kıs sistemas biológicos: «La lógica clásica, para la que sólo puede existir lí no contradicción, es inaplicable a la biología, donde se da una coexistencia constitutiva y una continuada lucha (que es, al mismo tiempo, sinergía) entre lo homogéneo y lo heterogéneo, con dominio de este último bajo pena de muerte. La lógica clásica [...] es una lógica inversa de la lógica de la vida, es una lógica de la muerte.»32

La necesaria referencia a la heterogeneidad (contratia a la homogeneización entrópica de los sisterras cetrados) es algo inherente a los sistemas abiertos, abocados a procedir, por razones de «supervivencia», a una selección progresiva de alternativas o reducción de la complejidad del entorno. La dinámica del ineluclible intercambio sistema-entorno redefine siempre ex novo los parámetros iniciales, de modo que el tiempo mismo «se concibe como dimensión dell sistema y no como parámetro ontológico [...] no siendo la estructura y el cambio conceptos contradictorios, al igual que no lo son los de estabilidad y cambio. Es interesante, al respecto, el papel primordial que juega aquí la memoria del sistema, que impide la caída del mismo en la exclusiva dependencia del flujo de información inmediatamente próximo. Se expresa así la gran significación que tiene la dimensión histórica de los sistemas sociales de cara a su independencia y potencial de vigencia [...] Como indiscutible elemento constitutivo del sistema, el tiempo debe considerarse como categoría fundante de su identidad y no como peligro para la mismas.

31. N. Luhmann, «Funktionale Methode und Systemtheorie», Soziale Welt is (1964), pp. 1-25.

32. F. Samaranch, «Energia y ibernética», en S. Lupasco: Logique et contradiction (París: FUP, 1974).

33. P. Waldmann, «Zeit und Yandel als Grundhestandteile sozialer Systems», en Koslner Zeitschrift 23 (1971), pp. 687-703. 
Por otra parte, la identidad es procesual-morfogénica más que motfostática: es decir, referida, en los sistemas socioculturales, a los mecanismos propios de continua ampliación de la desviación respecto de los parámetros originatios. ${ }^{34} \mathrm{La}$ reciproca interacción de variables va modificando continuamente las nuevas bases de la estructuración de forma que la multiplicidad de interacciones imposibilita predecir, at margen de los procesos, cuáles serán los efectos. La causalidad clásica, concebida en un marco estático y equifinalista (en el sentido de qué condiciones iniciales similares desartollan estados finales similares), se redefine drásticamente desde una perspectiva multifinalista (en el sentido de qué condiciones iniciales similares desarrollan situaciones disímiles finales o qué condiciones iniciales disímiles pueden desembocar en resultados similares). M. Maruyama observa que la explicación del estado final debe basarse en «los procesos causales mutuos de retroalimentación positiva amplificadores de la desviación» más que en las condiciones iniciales.

Frente a la formulación clásica de teorías de la difusión, evolución, aculturación, moderaización, etc., el nuevo enfoque sistémico trata la desviación y el cambio explícitamente como proceso dialéctico de variables que interactúan y redefinen (amplificándolas o teduciéndolas) Ias condiciones iniciales: por ejemplo, fomentando «el desartolio del subdesarrollo» (A. G. Frank) y desarrollando «acumulaciones» (de poder, capital, etc.), "ćrtculos viciosos» (de discriminación, pobreza, etc.), de la desviación, que hacen tealidad, de algún modo, el postulado de la «profecía que se cumple a sí misma». Utilizando explícitamente la teoría de los sistemas, Leslie $\tau$. Wilkins estudia la desviación de los drogadictos en función de la reacción más o menos tolerante de la sociedad, así como de la vetificación de un circuito de retroalimentación de información: la estructuración de la desviación y de la conformidad y su amplificación o reducción dependen de una red de transacciones y diversas intransigencias entre grupos. ${ }^{35}$ Parecida estrategia ha seguido Lewis A. Coser en la explicación de «las funciones de la desviación y de la flexibilidad normativa». ${ }^{36}$

Doctrinas clásicas de Ia desviación, tales como la del comportamiento anómalo como fenómeno «normal» (Durkheim), de la «asociación diferencial» (Sutherland) y de la correspondiente (en el sentido de Sutheriand)

34. Magorch Maruyama, «The Second Cybernetics: Deviation-Amplifying Mutual Causal Processesm, en W. Buckley, op. cit., pp. 304-317.

35. L. T. Wilkins, Social Deviance (Englewood Cliffs: Prentice-Hall, 1965).

36. L. A. Coser, some Functions of Deviant Behavier and Normative FlexibiJityw, American Journal of Sociology 68 (1962), pp. 172-181 
ampliación de las teorías durkheimiana y mertoniana de la anomía (por ejemplo, R. Cloward, que, partiendo de la «invisibilidad» de ciertos comportamientos, incluye explícitarrente la referencia del acceso a la estructura de medios ilegítimos) har encajado progresivamente en el nuevo enfoque interaccionista y sistémico de teorías psiquiátrico-sociales (antipsiquiatría) de R. D. Laing, D. Cooper, T. Schaeff y T. S. Szasz, que tegistran muchos puntos en contacto corı la llamada labeling theory o teoría del etiquetamiento.

La desviación aparece, pues, como un proceso (más que como un estado) de etiquetamiento, en el sentido de que hay desviaciones que se amplifican (en función de factores, tales como clase social, campo-ciudad, etcétera), y otras que no se amplififican e incluso se reducen (el «desviado secreto» de H. Becker, el «delincuente de cuello blanco» de E. H. Sutherland), no entrando estas últimas a constituir necesariamente parte integrante de la estructura de roles (desviación secundaria): "Cuando una persona empieza a utilizar su conducta desviada o un rol basado en ésta como medio de defensa, ataque o ajuste a los problemas manifiestos o encubiertos creados por la consiguiente teacción de la sociedad, su desviación es secundaria.. ${ }^{37}$ En parecido marco interaccionista simbólico y sistemático, E. Goffmann, ${ }^{38}$ aunque sea consciente de que son los actores los que van «elaborando el sistema», ha exp zesto magistralmente los procesos de «despojo del rol», «mortificación del yo», etc., en el marco sistémico de las tlamadas «instituciones totales» y de sus relaciones con el exterior. Se estudia, pues, la desviación más illá de la consideración estática (funcionalismo tradicional) o individual 'psicoanálisis freudiano), incluyendo explícitamente un tratamiento procesal y social en el que «la enfermedad mental es un mito, cuya función consiste en enmascarar y transformar en algo más aceptable la amarga pílclo:a de los conflictos morales presentes en las relaciones humanas». ${ }^{39}$ Gof:mann ha referido aquella a «ausencias», «participaciones ocultas» tealizadas por personas no autorizadas y en contextos «indebidos». Así, la «a'sencia» de un grupo en una función religiosa «convenientemente institucionalizada» (convencionalmente) no se la define como enfermedad (por ejemplo, esquizofrenia), mientras que otras ausencias son tenidas como sírtomas de la enfermedad mentâl. Algunas

37. E. Lemert, Social Patology (Nueva York: McGrown Hill, 1951), p. 76.

38. E. Goffmann, Internados Buenos Aires: Amortortu, 1970).

39. T. S. Szasz, «The Myth cf Mental Illness», en American Psychologist 15 (1960), p. 115.

40. E. Goffman, Bebavior in I'ublic Places (Nueva York: Free Press, 1964). 
investigaciones —así, la de J. Marsholl Townsend - han cotroborado esta correlación entre "concepciones populares» y «sintomatología», aunque se baya puesto en duda la especial eficacia atribuida a los estereotipos en la formación del síntoma. En general, queda en pie la tesis de que la sintomatología de la enfermedad mental, como «transgresión de reglas residuales $»^{42}$ debe comprenderse y explicarse en función del contexto social en que se producen. La misma intervención del psiquiatra $-\mathrm{y}$, correlativamente, del juez- y el correspondiente diagnóstico fallo «es un paso fundamental que organiza $y$ activa la reacción de la sociedad $\gg,{ }^{43}$ siendo, en general definido como desviado -mental o no-- aquel o aquellos grupos a los que se (a través del precipitante de los «empresarios motales») haya aplicado con éxito dicho rótulo. Así, la desviación social es "parte de una actividad social», es la actividad social misma [Albert K. Cohen: Des* viance and Control (Englewood Sliffs: Prentice-Hall, 1966) Cap. 9].

Desde una perspectiva igualmente sistémica e interaccionista he enfocado yo mismo cruciales aspectos de la desviación en la estructura social de España. Aśi, se ha utilizado el dato de la mayor tasa -al menos, con un peso relativo sensiblemente superior al observado en otros países eu* ropeos- de suicidios en el campo respecto de la ciudad como indicador de un sistema de retroalimentación positivo/negativo (anomía ambivalente) en el que la fuerte emigración refleja el intento de superación de un con* flicto entre modos de producción diferentes, en el seno de una misma formación social, y, consecuentemente, entre sistemas de valores, notnas y comportamientos diferentes. Los procesos han sido únicamente insinuados y tequieren, naturalmente, un análisis más detallado. El modelo sistémico. analítico me ha ayudado a descubrir y explicar, al mismo tiempo, los momentos de ritualismo de los comportamientos del campo y del emigrante. En consecuencia, el conformismo - y también el fatalismo-m son más aparentes de lo que normalmente -es decir, desde perspectivas estáticas-- se piensa y se ha pensado desde que, incluso desde posiciones maxxistas, se ha ignorado la capacidad de cambio, incluso revolucionario, de las es. tructuras rurales. Las perturbaciones procedentes del entorno (mundo ur-

41. J. M. Townsend, «Cultural conceptions, mental disorders and social roles: A comparison of Germany and Anericam, American Sociological Review 40 (1975), pp. 739.752 .

42. Th. Scheff, El rol del enfermo mental (Buenos Aites: Amorrortu, 1974), pp. 38 y ss.

43. Th. Scheff, op. cit., p. 121. 
bano) tienden a registrarse en el sistema considerado (campo, emigrante) como imposiciones, provocaciores, etc., que generan una reelaboración de estructuras siguiendo incluso la vía de la violencia. Esta circunstancia se aprecia en la mayor carga violenta en la estructura delictiva del campo y del emigrante, pese a que sus delitos tegistren a menudo una frecuencia relativa menor que los de la ciudad o de la sociedad anfitriona. $\mathrm{La}$ apuntada violencia puede concebirse como el producto de retroalimentaciones (pro ducto reprimido) ciudad-campo. Sucede que «la crisis del campo es factor de la emigración, que, a su ve;; contribuye, de rebote, a agtavar aquélla. La situación especialmerte anónica del campo español explica el sorprendente hecho de que éste supere actuaimente a la ciudad en su tasa de suicidios». ${ }^{44}$ Esta circunstancia, que hasta cierto punto podría parecer normal dada la peculiar estructura de edades del campo español (más vieja y, por lo mismo, más proclive al suicidio), sitúa a España en una situación especial dentro del contexto europiso-occidental, incluso controlando el factor edad.

La explicación del fenómero apuntado tiene, pues, sentido en el marco de un análisis sistémico basado en la interacción, con retroalimentaciones más bien positivas, de las siguientes variables: u) urbanización, m) modernización-industrialización, a) anımía, e) emigración). Cada una actuaría sobre la siguiente y la última alimentaría la primera cerrando el circuito.

Como se ha indicado, el enfoque sistémico cuadra con el esquema de la teoría del etiquetamiento cono alternativa al enfoque estructural-funcional-tradicional. Este ha visto el orden social como resultado de socializaciones compartidas, sin tener suficientemente en cuenta que el orden social es siempre problemático por su condición de negociado y legitimado (interpretado) en la práctica cotidiana (etnometodología): Así, «se considera el orden social no como producto de fuerzas externas (absolutas) a los individuos, sino más bien como resultado de construcciones intencionadas del orden social en el que los significados morales y problemáticos se consideran parte de los recursos simbílicos requeridos en la construcción exitosa del orden de nuestra sociedad $\%{ }^{45}$ Se ve, pues, cómo el enfoque sistémico analítico puede encajar la teoría del etiqueramiento y las nuevas contribuciones de corrientes, tales cora la etnometodología, como se apuntará luego.

44. J. A. Garmenđia, Esquemo del delito en Españo (Batcelona: Plaza y Janés, 1974), p. 94. p. 8 .

45. J. D. Dougtas, Deviance and Respectability (Nueva York: Basic Books, 1970), 


\section{Estructura social:}

procesos de institucionalización y su relación con las ideologias

Lo estructural no se reduce, pues, a lo normativo, a la cultura normativa institucionalizada, que, desde un análisis profundo, aparece como producto del juego de fuetzas o simple «modelo consciente» (Lévi-Strauss): es decir, el sistema institucional no refleja sino de modo imperfecto la estructura social. Sin compartir necesariamente el conocido enfoque de N. Poulantzas, cabe aceptar la siguiente observación: «Estructura-instittsción: deben distinguirse bien estos dos conceptos. Se entenderá por institución un sistema de normas o de reglas socialmente sancionado [...]. Por el contrario, el concepto de estructura comprende la matriz organizadora de las instituciones. Por el funcionamiento ideológico, la estructura siempre permanece oculta en -y por- el sistema institucional que ella organiza, $\rangle^{46}$

Las anteriores puntualizaciones implican necesariamente una referencia a la ideología en el estudio de la institución, incluida la de la sociología y de sus usos. Desde la perspectiva de una «sociología de la sociología», «sociología teflexiva», «etnometodología» (H. Garfinkel), etc., la sociología misma, como institucionalización del saber acerca de lo social debe enmatcarse, para su explicación y también aplicación, en la estructura social: precisamente, el cuestionamiento radical de ésta - o sea, una pregunta más profunda sobre ella - ha sido, al mismo tiempo, un importante momento en la génesis y desarrollo de la sociología. Esta disciplina ha seguido diversas peripecias, y las más prometedoras no parecen haberse registrado precisamente cuando aquel cuestionamiento ha sido institucionalizante $y$ reifcante. El consensus normativo, que priva a menudo en el análisis institucional, pasa por alto los aspectos conflictivos y negociadores del mismo entre fuerzas sociales diversas.

Desde las perspectivas apuntadas, la institucionalización aparece cono proceso siempre inestable o resultado inseguro de una negociación, acompañada siempre de alguna imposición, entre partes y grupos de la estrustura social en «desarrollo desigual» ${ }^{47} \mathrm{El}$ consensus normativo institucional alcanzado en cada momento aparece, pues, como algo tenue y siempre revisable. Desde el punto de vista del análisis de sistemas, el orden institucional se concibe como «recreándose» continuamente, presidido por re-

46. N. Poulantzas, Poder foltitico y clases sociales en el Estado capitalisto (Madrid: Siglo XXI, 1972), p. 140.

47. G. Lapassade, Las claves de la sociología (Barcelona: Laia, 1973), pp. 189-223. 
troalimentación negativa (que f́vorece la estabilidad) y positivas (que contribuyen a la elaboración de nuevas estructuras y sitúan el sistema en niveles diferentes), de forma que los estados finales aparecen no sólo como resultado de las condiciones iniciales sino de la recíproca interacción de variables o «procesos causales mutuos de amplificación o reduzcción de la desviación» (M. Maruyama). Lcs procesos no siguen, pues, una trayectoria determinista y lineal.

La sociología, no sólo la marxista, abunda en este enfoque necesariamente conflictivo y dinámics de los procesos de institucionalización: así, G. C. Homans ${ }^{48}$ recordó la imposibilidad de que las normas cubrau toda la regulación de la condurta, haciendo referencia explícita al correspondiente desatrollo subinstitucional capaz de sustituir el orden institucional vigente; Peter Blau ${ }^{48}$ Ilamiz la atención a los momentos de transformación dialéctica del cuadro institucional desde el intercamabio social, el juego del poder y el proceso die legitimación; David Easton ${ }^{50}$ estudia la conversión de deseos en demandas dentro del sistema político y de su entorno; R. H. Turner ${ }^{51}$ analiza los procesos morfogénicos de "asunción de toles» $\mathrm{y}$, en consecuencia, de la institucionalización o proceso de «resourción de la tensión del rol» (Gıode); Nadel ${ }^{52}$ apunta a la insuficiencia cée las normas en la explicación de la estructura social si no se tiene en cuenta el cálculo de los riesgos de la expectativa del éxito.

Lo apuntado reclama la necesidad de considerar la institucionalización como un proceso inseparable de la desviación. Por lo mismo, debe darse siempre por supuesta la presencia del poder $P=p_{1}-p_{2}$, donde $p_{1}$ representa la probabilidad de que $P$ ejecute las instrucciones de $A$, mientras que $\mathrm{p}_{2}$ simboliza la probabilidad de una ejecución motu propio (R. A. Dałl: «The concept of power», en Bebavioral Science, 2, 1975, p. 106): es decir, el poder condiciona la actoridad (concebida como "poder legítimo») y ha de saltar en su auxilio, dado que no es fácil la existencia de un sistema de dominación plenamente legitimado o de consensus normativo. ${ }^{53}$

48. G. C. Homans, Social Beinavior: Its Elementary Forms (Nueva York: Harcourt Brace, 1961).

49. Peter M. Blau, Exchange and Power in Social Life (Nueva York: John Wiley, 1964).

50. David Easton, A Framework of Political Analysis (Englewood Cliffs: Prentice-Hall, 1965).

51. R. H. Turner, «Role-Taking», en A. Rose: Human Bebavior and Social Processes, cap. II.

52. S. F. Nadel, «Social Control and Self-Regulation», Social Forces 31 (1953), pp. $256-273$.

53. Cf. J. A. Garmendia, «Pcder», en Diccionario..., op. cit., p. 513. 
Aparece así la estructura institucional como permanente colaboración por parte de los protagonistas de la negociación (intento de dominio) y, en última instancia, de la actividad humana. Se la concibe, pues, como producto que, como tal, tiende a cristalizar en moldes bien perfilados. Los mismos actores reifican fácilmente comportamientos que comportan habituación, tipificación, rutinización o control en base a la asunción recíproca de roles. Sin embargo, por lo que tiene de negociación, la institucionalización tiende, por otra parte, a descosificarse. Por lo mismo, la institución necesita, para conservarse, de cierta legitimación frente a un mundo de actores no «supersocializados». Se ha dicho que la lógica institucional «no reside en las instituciones y sus funcionalidades externas sino en la manera como éstas son tratadas cuando se reflexiona sobre ellas. Dicho de otro modo, la conciencia reflexiva superpone la lógica al orden institucional [...]. La «lógica» que así se atribuye al orden institucional es parte del acopio de conocimiento socialmente disponible (de forma que) la integración de un orden institucional puede entenderse sólo en términos del «conocimiento", ${ }^{54}$ entendido éste como el propio de la reflexión generalizada, que incluye también (pero no exclusivamente) el conocimiento especializado del sociólogo.

En este contexto encaja perfectamente la afirmación de que «la sociología del conocimiento se ocupa del análisis de la construcción social de la realidad». ${ }^{55} \mathrm{La}$ sociología del conocimiento debe investigar, pues, no sólo el conocimiento «científico», sino el conocimiento «no científico» o precientífico propio de la estructura del sentido comín (A. Schuetz) de las distintas instancias negociadoras de la institución. ${ }^{36}$ En tal sentido, "el abandono del método puede ser una condición necesaria para la mejora de nuestro conocimiento y de nuestras vidas». ${ }^{.7}$ Se construye (y legitima) y destruye (deslegitima) un orden institucional en función de un forcejeo dialéctico entre «conocimientos» (reflexiones, interpretaciones, ideologías, ciencia) diversos de lo social, que, por otra parte, aparecen como prácticas enraizadas en una estructura social. La pretendida desideologización supondría, pues, la afirmación de un orden estático. Ello implicaría una completa coincidencia de los «conocimientos» disponibles, que, entre otras cosas, comporta la tesis de una conciencia social axiológicamente neutral: así, desde bases opuestas a la conocida tesis del final de las ideologías,

54. P. L. Berger y Th. Luckmann, La construcción social de la realidad (Buenos Aires: Amorrortu, 1968), p. 88.

55. Ibid.

56. A. Schuetz, Der sinnbafte Aufbau der sozialen Welt (Viena: Springer, 1960).

57. Derek L. Phillips, Abandoning method (Londres: Jossey-Bass, 1973), p. 151. 
Adam Schaff ${ }^{58}$ se niega a contraponer ciencia e ideología, en el sentido más común de este término, porque ello supondría admitir la posibilidad de una ciencia desprovista de juicios de valor. Para A. Schaff, la ideología es simplemente «un sistema de opiniones» - se excluye la caracterización como deformada o no- que puede ser científico o correcto (ideología científica) y lo es en el caso del marxismo, encarnado en el proletariado y su conciencia histórica (Lukács). En el fondo subyace la creencia de haber descubierto finalmente la estructura oculta, al ser bajo las apariencias (por ejemplo, las relaciones abstracto-formales) del capitalismo.

Por su parte, L. Althusser considera la ideología como una «representación de la relación imaginaria de los individuos con sus condiciones reales de existencia»: ${ }^{59}$ es decir, las condiciones reales o estructuras son ocultas al pensamiento ideológico y es la ciencia la que debe ocuparse de investigar la estructura. Se ha visto en esta posición una mutilación del papel que la lucha de clases tiene en la génesis de la deformación ideológica, siendo aquél más bien suplementario o «sobredeterminante» en una sociedad capitalista. ${ }^{60}$. De todos modos, aparece bien claro en Althusser el «corte epistemológico» entre ideología y ciencia.

Los obstáculos ideológicos al conocimiento científico han querido ser soslayados desde otros puntos de partida por K. Mannheim (Ideología y utopía. Madrid: Aguilar, 1958), que propuso una formulación general de la ideología, según la cual estarían deformados todos los «conocimientos» aisladamente (desde la propia perspectiva) considerados, dada la constitutiva «determinación existencial del pensamiento» (Seinglebundendenbeit des Denkes), imponiéndose el relacionismo como solución de objetividad, especialmente asequible a los intelectuales (freiscbwebende intelligenz). Es una postura diferente a la de Max Scheler, para quien los «factores reales» sólo determinan la existencia (Daseis) pero no el contenido (Sosein) de los *factores ideales». ${ }^{61}$

De cualquier modo, el descuido del aspecto organizacional y negociador (entre instancias, «conocimientos», etc.), de la estructura social propicia el dogmatismo y la tesis del final de las ideologías. Desde la perspectiva de la razón holística totalizadora, incluso bajo la autocalificación

58. A. Schaff, Sociología e ideología (Barcelona: A. Redondo, 1969).

59. L. Althusser, «Idéologie $t$ appareils idéologiques d'état», La Pensée, 151 (1970), p. 25.

60. M. A. Quintanilla, «Sobre el concepto marxista de ideología», Sistema 7 (1974), pp. 29 y ss.

61. Mención sobre Max Scheler, en P. L. Berger y T. Luckmann, op. cit., p. 22. 
«Papets»: Revista de Sociología

de dialéctica, se relegan a un segundo plano los elementos de negociación y lucha propios de la sociedad concebida fundamentalmente como organización más que como sustancia, como proceso más que como resultado.

\section{Conclusiones}

He intentado exponer en qué medida el estudio de la estructura social desde la perspectiva de los procesos supone una superación de teorías clásicas. Es una superación (Aufbebung) que arranca, superándolo luego, del primitivo énfasis del mismo funcionalismo (al igual que del estructura. lismo) en la relación (función) u organización frente al tradicional enfoque sustancialista. La apuntada superación ha sido gradual. En efecto, las primeras versiones del análisis estructural-funcional están teñidas todavía de sustancialismo al preferir la concepción de la sociedad como sistema cerrado al que accidentalmente sobrevendría el cambio. Finalmente, se ha mostrado cómo el tratamiento de lo sociocultural como sistema abierto se combina satisfactoriamente con teorías, tales como las del etiquetamiento, etnometodología, etc.

José A. GARMENDIA

Facultad de Ciencias Económicas

Universidad de Málaga

Málaga 